\title{
Marfan Syndrome with Varieties of Clinical Features
}

\author{
GOUTAM KUMAR ACHERJYA, ${ }^{1}$ KEYA TARAFDER, ${ }^{2}$ MD. DIN UL ISLAM,${ }^{3}$ MAHABUBUR RAHMAN, ${ }^{4}$ MOSTOFA \\ KAMAL CHOWDHURY5
}

\begin{abstract}
:
Abstract: Marfan Syndrome is an autosomal dominant disorder caused by mutation in the extracellular matrix protein fibrilin-1 gene located on chromosome 15. It has variable range of expression. We describe a case of a 12 year aged girl with positive family history including lean and thin body stature, skin striae, joint hyper mobility, high arched palate, mal occlusion of teeth, pectus excavatum, winging of scapula, scoliosis of back bone, total arm span more than total body height (1.11:1), lower segment greater than upper segment (1.40:1), severe form of mitral valve prolapse and aortic root dilatation. There are a lot of features of Marfan Syndrome in our single patient which is rare in earlier available case reports.
\end{abstract}

Key Words: Marfan Syndrome, Mitral valve prolapse, Dilatation of ascending aortic root.

\section{Introduction:}

Marfan syndrome is a heritable disorder of the connective tissue with an estimated prevalence of 1 in 5000 individuals and no predilection for either sex. The syndrome is inherited as an autosomal dominant trait with complete penetrance but with phenotypic expression that varies considerably, both between and within families. About $75 \%$ of persons with classic Marfan syndrome have a positive family history. Affected individuals develop varying patterns of organ involvement including the cardiovascular, ocular, skeletal, lung, skin and the dura. In classical Marfan syndrome, many manifestations present during puberty or later and severe complications rarely develop before adulthood. Such complications include severe scoliosis or pectus excavatum, spontaneous pneumothorax, retinal detachment or sight-threatening glaucoma resulting from dislocated lenses. ${ }^{1}$ Further less specific features including high arched palate, crowding of the teeth and skin striae. ${ }^{2}$ Before the evolution of open heart surgery, however, Marfan patients usually died from acute aortic dissection or

1. Junior consultant (Medicine), UHC, Bagerpara, Jessore, Bangladesh.

2. Lecturer, Department of Microbiology, Jessore Medical College, Jessore, Bangladesh.

3. Associate Professor, Department of Microbiology, Jessore Medical College, Jessore, Bangladesh.

4. Assistant Professor, Department of Pediatrics, Jessore Medical College, Jessore, Bangladesh.

5. Assistant Registrar, Department of Endocrinology, Dhaka Medical College Hospital, Dhaka, Bangladesh.

Corresponding author: Dr. Goutam Kumar Acherjya, Jonior consultant (Medicine), CHC, Bagergara, Jessore, Bangladesh, Email: gacherjya@hotmail.com rupture, and thus had an average life-expectancy of only 32 years. ${ }^{1}$ Medical management may not reverse the features seen, but can reduce the progression and the severity of the symptoms. Beta blockers are considered as the standard therapy, as they work by reducing the aortic shear stress and the heart rate. Diagnosis is mainly done using the Ghent criteria and a detailed clinical examination. ${ }^{3}$

\section{Case Presentation:}

Our index patient, 12 year aged girl, hailing from Bagerpara, Jessore, Bangladesh was admitted with the complaints of intermittent fever for 15 days and productive cough for same duration. On query, her mother stated that she had remained well up to 5 years of her age then she was growing up a little bit taller and thinner than other children of same age. There were occurring some abnormalities of her body shape with prominence of scapular region during supporting her hands. Her menarche started at the $9^{\text {th }}$ year of her age and it is now normal in flow and rate. She was immunized as per EPI schedule. Her mother looks lean and thin like our patient. Her father, two sisters and one brother have normal body shape.

Physical examination revealed that she looked lean and thin (Fig no. 1) with abnormal body shape. Her pulse was 96 beats/minute with normal rhythm and blood pressure 105/65 mm of Hg. She had malocclusion of teeth (Fig no. 3 ) and high arched palate (Fig no. 2) and pectus excavatum. Skin striae are present in the both anterior axillary folds and lower abdomen. Her joints were hyper mobile (Fig no. 05). There were thumb and little finger grips more than her wrist (Fig no.7) and winging of her left scapula and scoliosis of her back bone (Fig no. $04 \&$ 08). Steinberg sign was positive in our patient (Fig no. 06). The total height was 
$154 \mathrm{~cm}$, arm span is $171 \mathrm{~cm}$. The ratio of total arm span and total height was 1.11: 1. Her lower segment length from pubic symphysis to heel was $90 \mathrm{~cm}$ and upper segment from vault to pubic symphysis was $64 \mathrm{~cm}$. The ratio of lower segment to upper segment waas 1.40:1. Her cardiovascular system examination revealed that $1 \mathrm{st}$ and $2^{\text {nd }}$ heart sound appeared normal and presence of late systolic murmur in the left $3 \mathrm{rd}$ and $4^{\text {th }}$ intercostal space. Other systemic examination including eye were normal. Investigation findings of our patient are provided in Table no. 1.

Her mother showed joint hypermobility, wrist sign and Steinberg sing (Fig no. 9). She denied investigating her further.

\section{Discussion:}

The classical view of Marfan pathogenesis is that mutant fibrillin-1 molecules alter microfibril assembly in a dominant-negative manner. ${ }^{4}$ The classical paradigm

\section{Table-I}

Investigation profile of index patient

\begin{tabular}{ll}
\hline Name of investigation & \multicolumn{1}{c}{ Findings } \\
\hline CBC & $\begin{array}{l}\text { TC-9000/cmm, N-56\%, L-32\%, } \\
\text { E-09\%, Platelet count- } \\
204000 / \mathrm{cmm}, \text { ESR-45mm in } \\
1 \text { st hour, Hb-12.4\% }\end{array}$ \\
\hline Urine R/M/E & Albumin- Trace, \\
\hline PBF & Eosinophilia \\
RBS & 4.88 mmol/L \\
S. Creatinine & $0.81 \mathrm{mg} / \mathrm{dl}$ \\
CPK & 77 U/L \\
TSH & $\begin{array}{l}2.26 \\
\text { ECG }\end{array}$ \\
Inverted T wave in Inferior \\
Lead and Incomplete RBBB. \\
Chest x-ray \\
(Fig no. 08)
\end{tabular}

regards Marfan syndrome as the result of structural weakness of connective tissue. TGF- $\beta$ represents a group of cytokines that control cellular proliferation, cell cycle arrest, differentiation, apoptosis and matrix deposition. Sequestration of TGF- $\beta$ and control of its activation depends on binding of TGF- $\beta$ to latent TGF- $\beta$ binding proteins (LTBP), which in turn bind to fibrillin-rich microfibrils. A deficiency of fibrillin-1 therefore causes excess of active TGF- $\beta$, and studies on fibrillin-1-deficient mice documented increased activity of TGF- $\beta$, resulting in both inborn airspace enlargement and myxomatous cardiac valve disease. Moreover, both phenotypes were prevented by application of TGF- $\beta$ neutralizing antibodies. ${ }^{5}$ Thus, pharmacological manipulation of TGF- $\beta$ signaling may offer an option to treating Marfan syndrome and related disorders.

Marfan syndrome cannot be diagnosed by a single molecular test but requires a scoring system that combines various diagnostic items. The so-called Ghent nosology subdivides diagnostic features into "major criteria", "minor criteria", "organ involvement" and manifestations that only in combination with other manifestations constitute a "major" or "minor" criterion. Individuals without a family history of Marfan syndrome require major criteria in at least two different organ systems and involvement of a third organ system. Individuals carrying an FBN1 mutation known to cause Marfan syndrome or cases with a positive family history require one major criterion and involvement of an additional organ to establish Marfan syndrome. ${ }^{6}$ The classical standards imply: (1) counselling on lifestyle modification including moderate restriction of physical activity; (2) endocarditis prophylaxis; (3) serial imaging of the aorta; (4) $\beta$-blocker medication for aortic protection; and (5) prophylactic replacement of the aortic root. ${ }^{7,8}$

Our index patient has positive family history with lean and thin body stature, skin striae, joint hyper mobility, high arched palate, mal occlusion of teeth, pectus carinatum, winging of scapula, scoliosis of back bone, total arm span more than total body height (1.11:1), lower segment greater than upper segment (1.40:1), severe form of mitral valve prolapse and aortic root dilatation. There are a lot of features of Marfan syndrome in our single patient which is rare in earlier case reports.

Consent: Parents of our patient gave informed written consent for publication of the case report and accompanying pictures.

Conflict of interest: None. 


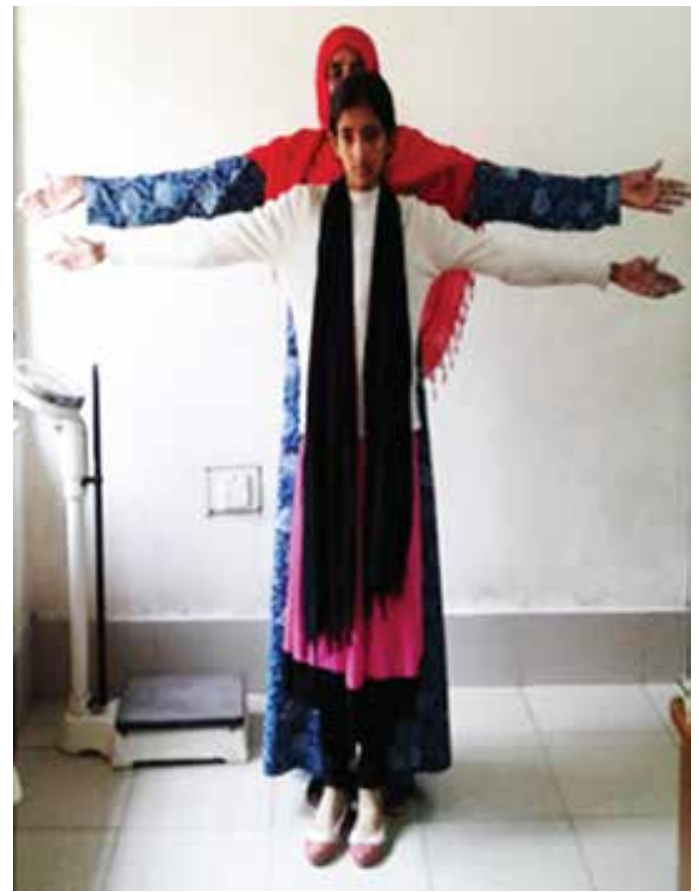

Figure 1: Lean and tall mother and our patient with long arm span



Figure 3: Malocclusion of teeth

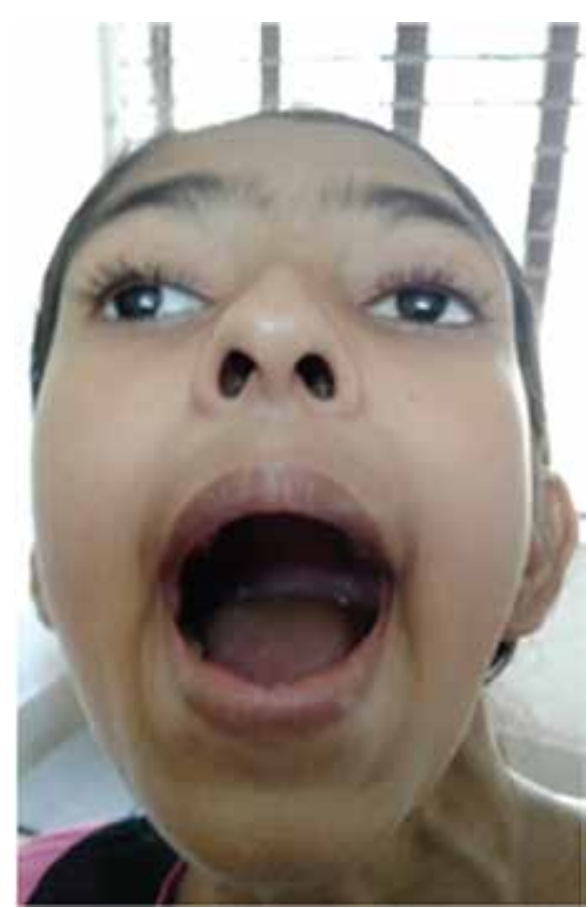

Figure 2: High Arched Palate

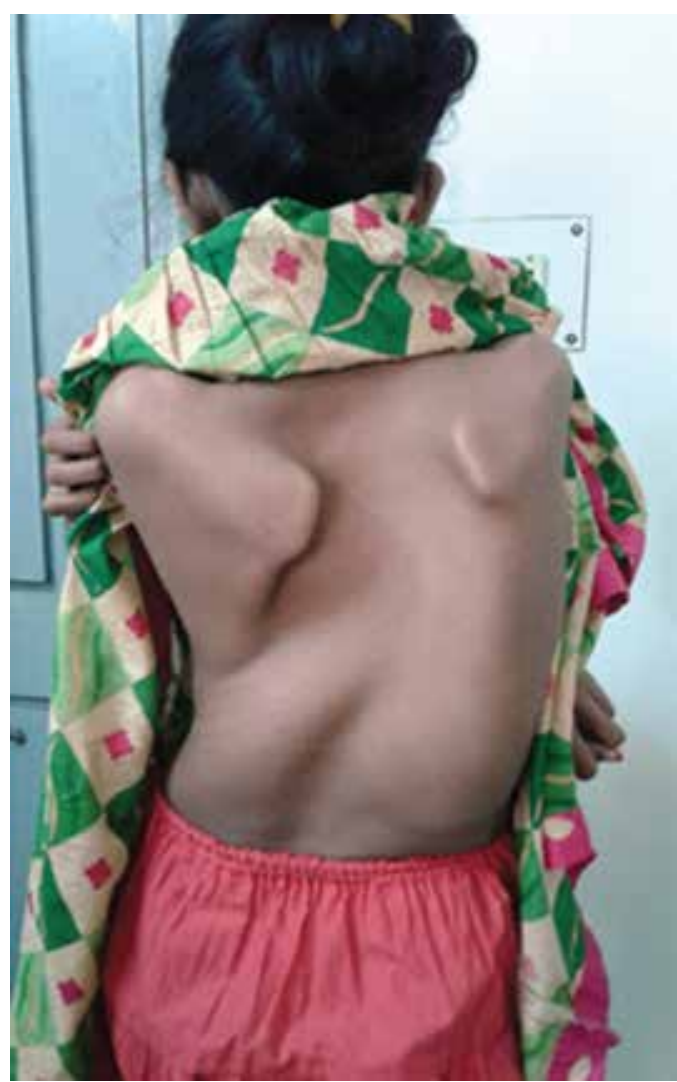

Figure 4: Winging of Scapula, Severe Dorsal Scoliosis 
JM Vol. 19, No. 1

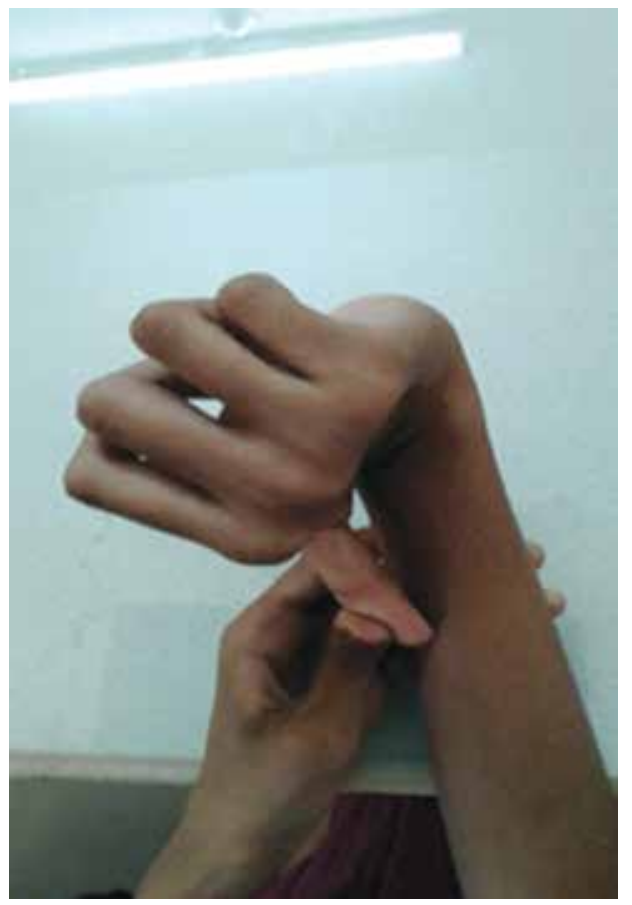

Figure 5: Joint Hypermobility

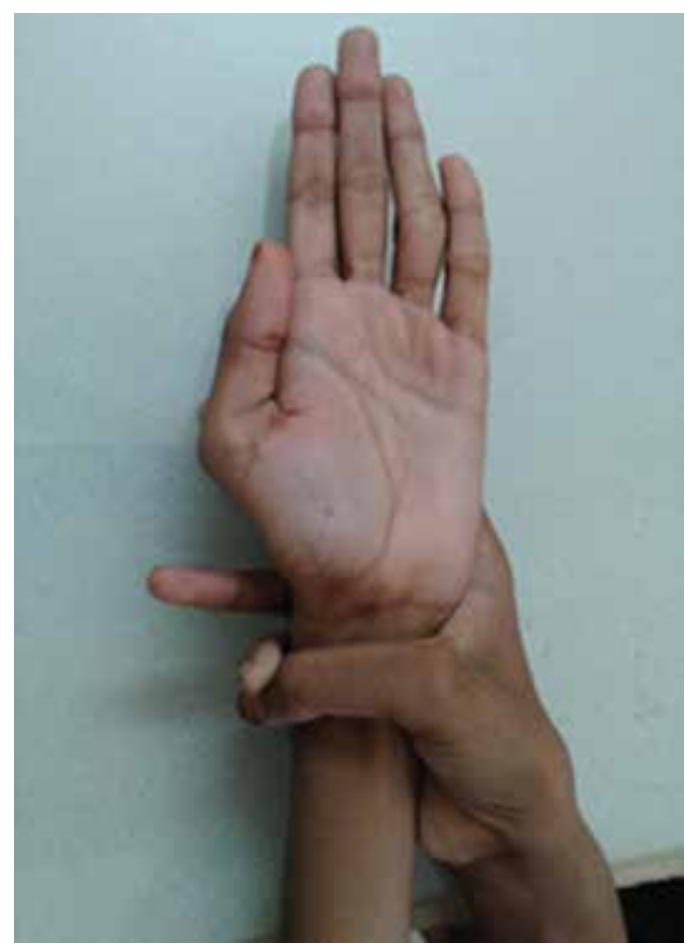

Figure 7: Wrist Sign
Marfan Syndrome with Varieties of Clinical Features

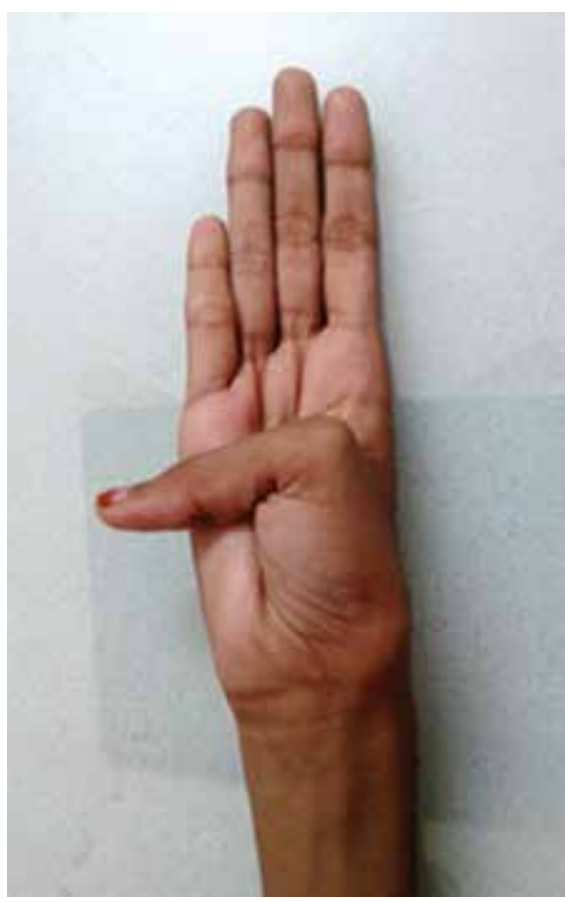

Figure 6: Steinberg Sign

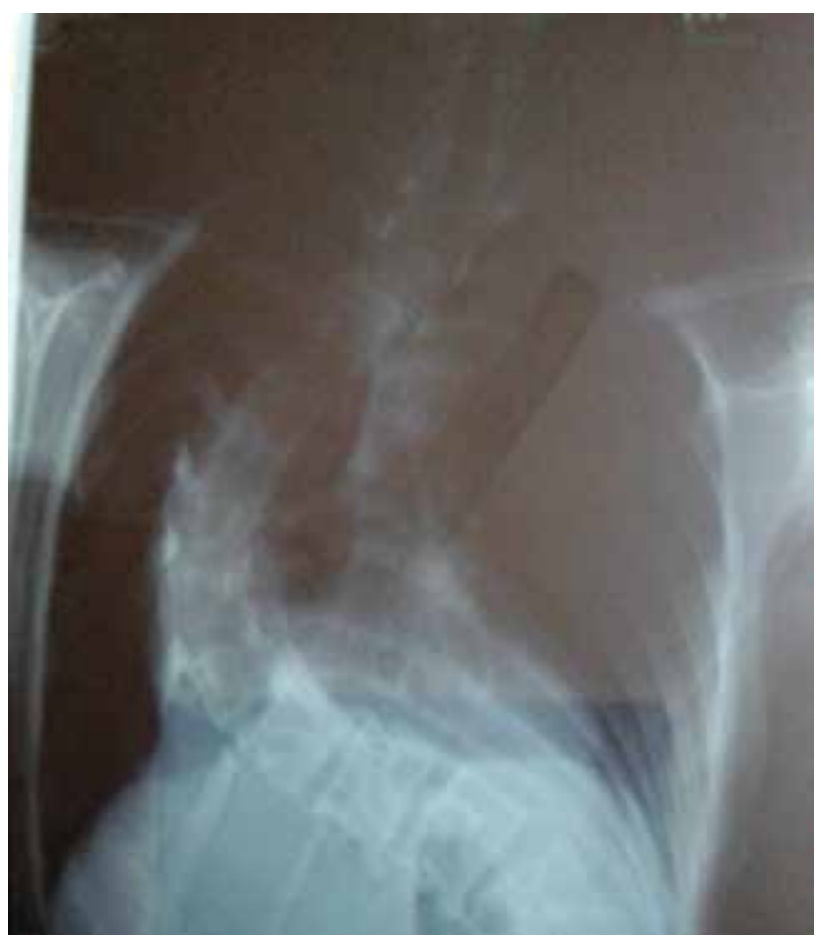

Figure 8: Chest x-ray (rib crowding and "S" shaped dorsal scoliosis) 

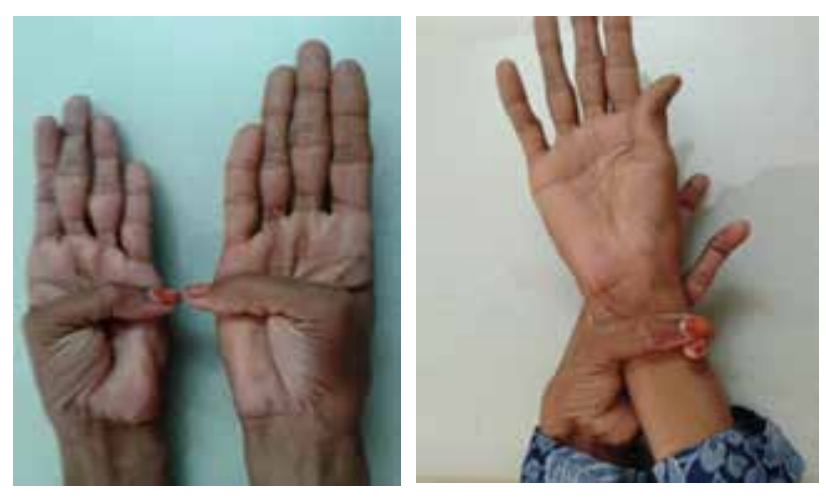

Figure 10: Patient's mother's Steinberg sign and Wrist sign

\section{References:}

1. Silverman D I, Burton K J, Gray J. et al Life expectancy in the Marfan syndrome. Am J Cardiol 1995;75:157-160.

2. Dean JC. Management of Marfan syndrome. Heart 2002;88(1):97-103.

3. Lacro RV, Dietz HC, Wruck LM, Bradley TJ, et al. Rationale and Design of a Randomized Clinical Trial of Beta Blocker Therapy (Atenolol) vs. Angiotensin Receptor Blocker Therapy (Losartan) in Individuals with Marfan Syndrome. Am Heart J. 2007;154(4):624-631.

4. Judge D P, Dietz H C. Marfan's syndrome. Lancet 2005;366:1965-1976.

5. Ng C M, Cheng A, Myers L. et al. TGF- $\beta$ dependent pathogenesis of mitral valve prolapse in a mouse model of Marfan syndrome. J Clin Invest 2004;114:1586-1592.

6. De Paepe A, Devereux R B, Dietz H C. et al. Revised diagnostic criteria for the Marfan syndrome. Am J Med Genet 1996;62:417-426.

7. Gott V L, Greene P S, Alejo D E. et al. Replacement of the aortic root in patients with Marfan syndrome. N Engl J Med 1999;340:1307-1313.

8. Von Kodolitsch Y, Rybczynski M. Cardiovascular aspects of the Marfan syndrome - a systematic review. In: Robinson PN, Godfrey M, eds. Marfan syndrome: a primer for clinicians and scientists. Eurekah.com and Kluwer Academic/Plenum Publishers 2004:45-69. 